4-1-1989

\title{
Equilibrium Polymerization on the Equivalent-Neighbor Lattice
}

Miron Kaufman

Cleveland State University, m.kaufman@csuohio.edu

Follow this and additional works at: https://engagedscholarship.csuohio.edu/sciphysics_facpub

Part of the Physics Commons

How does access to this work benefit you? Let us know!

Publisher's Statement

Copyright 1989 American Physical Society. Available on publisher's site at http://link.aps.org/ doi/10.1103/PhysRevB.39.6898.

Original Citation

Kaufman, Miron. "Equilibrium Polymerization on the Equivalent-Neighbor Lattice." Physical Review B 39 (1989): 6898-6906.

Repository Citation

Kaufman, Miron, "Equilibrium Polymerization on the Equivalent-Neighbor Lattice" (1989). Physics Faculty Publications. 1.

https://engagedscholarship.csuohio.edu/sciphysics_facpub/1

This Article is brought to you for free and open access by the Physics Department at EngagedScholarship@CSU. It has been accepted for inclusion in Physics Faculty Publications by an authorized administrator of EngagedScholarship@CSU. For more information, please contact library.es@csuohio.edu. 


\title{
Equilibrium polymerization on the equivalent-neighbor lattice
}

\author{
Miron Kaufman \\ Department of Physics, Cleveland State University, Cleveland, Ohio 44115
}

(Received 9 November 1988)

\begin{abstract}
The equilibrium polymerization problem is solved exactly on the equivalent-neighbor lattice. The Flory-Huggins entropy of mixing is exact for this lattice. We verify the discrete version of the $n$ vector model when $n \rightarrow 0$ is equivalent to the equal reactivity polymerization process in the whole parameter space, including the polymerized phase. The polymerization processes for polymers satisfying the Schulz distribution exhibit nonuniversal critical behavior. A close analogy is found between the polymerization problem of index $r$ and the Bose-Einstein ideal gas in $d=-2 r$ dimensions, with the critical polymerization corresponding to the Bose-Einstein condensation.
\end{abstract}

\section{INTRODUCTION}

The aggregation of monomers into linear polymers observed, for example, in sulfur, and various proteins exhibits critical phenomena effects. DeGennes and des Cloiseaux ${ }^{1}$ have observed that the polymerization process can be described by the high-temperature expansion of the $n$-vector model with $n \rightarrow 0$. This mapping was used to study the critical polymerization by means of the mean-field approximation, ${ }^{2} \epsilon$ expansion, ${ }^{3}$ and positionspace renormalization group. ${ }^{4}$ Gujrati $^{5}$ has pointed to peculiarities of the $n \rightarrow 0$ limit at low temperatures which may signal a phase transition line in the fugacities space of the polymer problem. Gujrati ${ }^{6}$ has also argued that mean-field theories of the $n \rightarrow 0$ model and of the polymer problem should not be equivalent at low temperatures. It is thus desirable to compare the $n \rightarrow 0$ vector model to an alternative exact solution of the corresponding polymerization model in the whole parameter space. One such comparison, one-dimensional polymerization, is already present in the literature. ${ }^{7}$ In this paper we obtain the exact solution of the polymerization problem on the equivalent-neighbor lattice. This lattice is a collection of $N$ vertices, each connected to the other $N-1$ vertices. Conventional spin models defined on the equivalentneighbor lattice are exactly solved by the mean-field theory. ${ }^{8}$

The polymerization process considered here is controlled by a fugacity $k$ conjugated to the number of chemical bonds and by a set of fugacities $\eta_{1}, \eta_{2}, \ldots$ conjugated to the numbers of polymers of size (number of bonds) $1,2, \ldots$, respectively. The polymerization problem studied by previous authors ${ }^{1-7}$ is obtained when all fugacities $\eta_{l}$ are equal to each other. In this case we verify its equivalence to the $n \rightarrow 0$ magnetic model in the whole parameter space, low-temperature regime included. Since the lattice under study here is the equivalent-neighbor one, the solution is closely related to the mean-field approximation, ${ }^{2,9}$ which in turn is equivalent to the chemical reaction theory of Tobolsky and Eisenberg. ${ }^{10}$

Flory ${ }^{11}$ has argued that the principle of equal reactivity, according to which the opportunity for reaction is independent of the size of the participating polymers, implies an exponential decay of the number of polymers of size $l$ as a function of $l$. This polymer number distribution is known as the most probable distribution. We reproduce this result when $\eta_{l}=\eta$, for all $l$ (i.e., consistent with equal reactivity). On the other hand, some experimentally measured polymer numbers as a function of the polymer size are described by the gamma distribution: exponential times power, also known as the Schulz ${ }^{12}$ distribution. Within our model the Schulz distribution is obtained, provided we abandon the equal reactivity principle and assume a power dependence: $\eta_{l}=\eta(l+1)^{r-1}$. The critical polymerization behavior depends strongly on the Schulz index $r$. If $r \geq-1$ (this includes the $r=1$ case of equal reactivity and the most probable distribution), the polymerized phase occurs at $\eta=0$ and $k \geq 1$. It contains a small number of macroscopically large polymers. If $r<-1$, the polymerized phase occurs for $k \geq k_{c}(\eta)$. It is a mixture of a small number of macroscopically large polymers and of a large number of microscopically small polymers. This is akin to the two-fluid model ${ }^{13}$ of superfluidity. The critical exponents change continuously with the Schulz index $r$. There exists a close analogy between the Schulz polymerization of index $r$ on the equivalent-neighbor lattice and the Bose-Einstein condensation in $d=-2 r$ spatial dimensions. The Stockmeyer et $a l .{ }^{14}$ theory of ring polymerization is also analogous to the Bose-Einstein condensation.

The balance of this paper is organized as follows. In Sec. II we derive the exact solution of the polymerization process on the equivalent-neighbor lattice. We find that the entropy associated with putting linear polymers on this lattice is closely related to the Flory-Huggins ${ }^{15}$ entropies of mixing and disorientation. In Sec. III we explicitly verify the equivalence of the polymerization process, with all the fugacities $\eta_{l}$ equal to each other, to the $n \rightarrow 0$ vector model for the whole parameter space. The polymerization process with fugacities $\eta_{l}=\eta(l+1)^{r-1}$ is analyzed in Sec. IV. A summary of results is found in Sec. V.

\section{MODEL AND SOLUTION}

We consider a lattice of $N$ vertices, each connected to the other $N-1$ vertices: This is the equivalent-neighbor lattice. Each vertex is occupied by one monomer. Chemical bonds can be established between monomers along 
the lattice bonds in accordance with the following rules. Between any two monomers there is at most one chemical bond. At most two chemical bonds meet at any monomer. Each polymer group of two or more monomers connected by chemical bonds, has two endpoints, each of valence 1 . The other monomers on the polymer have each a valence of 2 . This is a mixture of free monomers and linear polymers.

The partition function is

$$
Z=\sum k^{B} \eta_{1}^{P_{1}} \eta_{2}^{P_{2}} \eta_{3}^{P_{3}}, \ldots
$$

where $B$ is the total number of bonds, $P_{l}$ is the number of polymers of size $l$ (i.e., $l$ bonds and $l+1$ monomers). The fugacities $k$ and $\eta_{l}$ are positive quantities, to ensure the positivity of all probabilities. The sum in Eq. (1) is over all possible graphs of linear polymers and monomers, an example of which is shown in Fig. 1. The summation over graphs can be changed to a summation over the numbers of polymers $P_{1}, P_{2}, \ldots, P_{N-1}$ by introducing the combinatorial factor $\Gamma_{N}\left(P_{1}, P_{2}, P_{3}, \ldots, P_{N-1}\right)$,

$$
Z=\sum \Gamma_{N}\left(P_{1}, P_{2}, P_{3}, \ldots\right) k^{B} \eta_{1}^{P_{1}} \eta_{2}^{P_{2}} \eta_{3}^{P_{3}}, \ldots,
$$

The combinatorial factor in Eq. (2) counts the number of graphs with the same number of polymers $P_{1}, P_{2}, \ldots, P_{N-1}$.

We next calculate the combinatorial factor for the equivalent-neighbor lattice in three steps. First we consider 1 polymer of size $B$, i.e., $B$ bonds,

$$
\Gamma_{N}\left(0, \ldots, P_{B}=1, \ldots, 0\right)=\frac{1}{2} N ! /(N-B-1) !
$$

This formula is justified as follows. The number of ways of picking the two ends of the polymer is $\frac{1}{2} N(N-1)$. For a given pair of ends the number of linear paths connecting them is $(N-2)(N-3) \cdots(N-B)$.

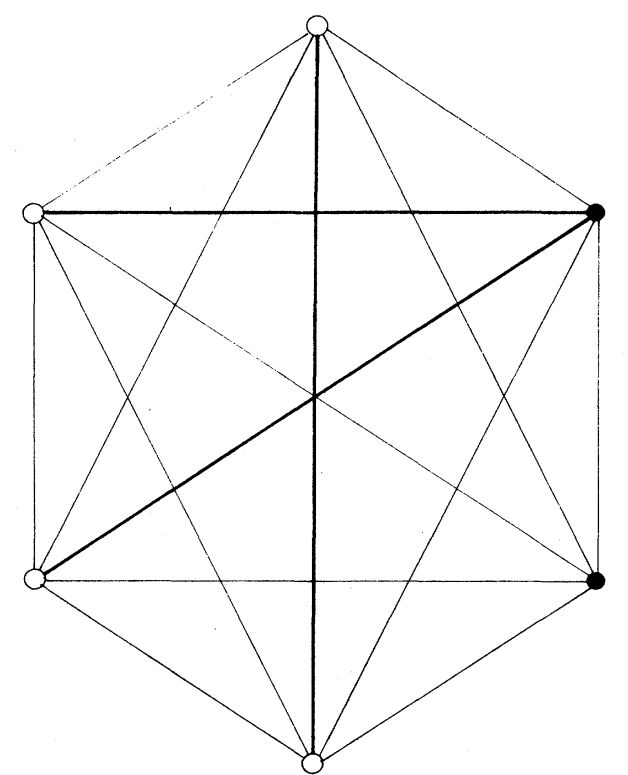

FIG. 1. Configuration of linear polymers on the equivalentneighbor lattice of $N=6$ vertices and 15 edges. Each vertex is occupied by a monomer. The heavy lines represent chemical bonds and the light lines represent unoccupied edges. Open circles represent monomers at polymers ends. There are $P_{0}=1$, $P_{1}=1$, and $P_{2}=1$ polymers and $B=3$ bonds. Since the lattice is embedded in a high-dimensional space there are no crossings, i.e., polymers are self-avoiding lines.

In the second step we count the number of ways of putting $P_{B}$ polymers, each of size $B$, on the equivalentneighbor lattice

$$
\Gamma_{N}\left(0, \ldots, P_{B}, \ldots, 0\right)=\frac{N !}{P_{B} ! 2^{P_{B}}\left[N-P_{B}(B+1)\right] !} .
$$

Equation (4) is obtained from Eq. (3) and

$$
\begin{aligned}
\Gamma_{N}\left(0, \ldots, P_{B}, \ldots, 0\right)= & \Gamma_{N}\left(0, \ldots, P_{B}=1, \ldots, 0\right) \\
& \times \Gamma_{N-B-1}\left(0, \ldots, P_{B}=1, \ldots, 0\right) \cdots \Gamma_{N-\left(P_{B}-1\right)(B+1)}\left(0, \ldots, P_{B}=1, \ldots, 0\right) / P_{B} !
\end{aligned}
$$

The factorial on the right-hand side of Eq. (5) is required by the indistinguishability of the $P_{B}$ polymers.

In the third step we calculate the number of ways of putting $P_{1}$ polymers of one bond each, $P_{2}$ polymers of two bonds each, . . , and $P_{N-1}$ of $N-1$ bonds each on the equivalent-neighbor lattice,

$$
\Gamma_{N}\left(P_{1}, P_{2}, P_{3}, \ldots, P_{N-1}\right)=\frac{N !}{P_{0} ! P_{1} ! P_{2} ! \cdots P_{N-1} ! 2^{P}}
$$

where $P=P_{1}+P_{2}+\cdots+P_{N-1}$ is the total number of polymers and $P_{0}$ is the number of free (not on polymers) monomers. Equation (6) is obtained from Eq. (4) and

$$
\begin{aligned}
\Gamma_{N}\left(P_{1}, P_{2}, P_{3}, \ldots, P_{N-1}\right)= & \Gamma_{N}\left(P_{1}, 0,0, \ldots, 0\right) \\
& \times \Gamma_{N-2 P_{1}}\left(0, P_{2}, 0, \ldots, 0\right) \cdots \Gamma_{N-2 P_{1}-3 P_{2}-\cdots-(N-1) P_{N-2}\left(0,0,0, \ldots, P_{N-1}\right)}
\end{aligned}
$$


The logarithm of the combinatorial factor given in Eq. (6) determines the entropy associated with arranging $P_{1}, P_{2}, \ldots$, and $P_{N-1}$ polymers on the $N$ vertices of the equivalent-neighbor lattice. This entropy can be split in two contributions: the Flory-Huggins entropy of mixing $^{15}$ and the disorientation entropy. ${ }^{16}$ The FloryHuggins entropy is extensively used in thermodynamic studies of polymer solutions as an approximate method for three-dimensional systems. The fact that it is also exact for a well-defined statistical problem ensures the laws of thermodynamics are satisfied, ${ }^{17}$ which may explain its considerable success.

Since each term in the sum on the right-hand side of Eq. (2) is of the type $\exp \left[N \Omega\left(p_{1}, p_{2}, \ldots, p_{N-1}\right)\right]$, where $p_{l}=P_{l} / N$, in the thermodynamic limit $N \rightarrow \infty$ the partition function $Z$ is ${ }^{8,18}$

$Z=\max _{\left\{P_{1}, P_{2}, \ldots\right\}} \Gamma_{N}\left(P_{1}, P_{2}, \ldots\right)(k / N)^{B} \eta_{1}^{P_{1}} \eta_{2}^{P_{2}} \cdots$

Note the added factor $N^{-B}$ to the right-hand side of Eq. (8) ensures the extensivity ${ }^{16}$ of the thermodynamic potential. The thermodynamic potential $f=-\ln Z / N$ is obtained from Eq. (8) after applying the Stirling formula

$$
f=\min _{\left\{p_{1}, p_{2}, \ldots\right\}} \Omega
$$

where

$$
\Omega=p_{0} \ln \left(p_{0}\right)+\sum_{l=1}^{\infty} p_{l} \ln \left(2 p_{l} / \eta_{l}\right)+b \ln (e / k),
$$

where $p_{l}=P_{l} / N, b=B / N$, and $p_{0}$ is the fraction of free (not on polymers) monomers. The minimization of $\Omega$ is performed with respect to $p_{1}, p_{2}, \ldots$ since $b$ and $p_{0}$ depend on the $p_{1}$ 's according to

$$
\begin{aligned}
& b=\sum_{l=1}^{\infty} l p_{1}, \\
& p_{0}+\sum_{l=1}^{\infty} p_{l}+b=1 .
\end{aligned}
$$

The minimization of $\Omega$ yields

$$
p_{l}=\frac{\eta_{l}}{2 k} x^{l+1}
$$

where $x=k p_{0}$. The total number of polymers per $N$ is then

$$
p=\sum_{l=1}^{\infty} p_{l}=\sum_{l=1}^{\infty} \frac{\eta_{l}}{2 k} x^{l+1}
$$

and the total number of bonds per $N$ is obtained from Eqs. (11) and (13),

$$
b=\sum_{l=1}^{\infty} \frac{\eta_{l}}{2 k} l x^{l+1}
$$

For given values of the fugacities the parameter $x=k p_{0}$ is determined by using the "equation of state," which is obtained from Eqs. (12), (14), and (15),

$$
k=x+\frac{1}{2} \sum_{l=1}^{\infty} \eta_{l}(l+1) x^{l+1} .
$$

Once $x$ is determined, $p$ and $b$ are obtained from Eqs. (14) and (15) and $p_{0}=x / k$.

The fraction of monomers belonging to polymers of $l$ bonds each is

$$
\Phi_{l}=(l+1) p_{l}=\frac{\eta_{l}}{2 k}(l+1) x^{l+1} \text { for } l>0
$$

and

$$
\Phi_{0}=p_{0}=x / k
$$

Assuming all monomers are identical, $\Phi_{l}$ is also the volume fraction occupied by polymers of $l$ bonds each. The thermodynamic potential $f$ given in Eqs. (9) and (10) can be expressed in a simpler form after using the equation of state, Eq. (16)

$$
f=\ln \Phi_{0}+b \text {. }
$$

The fluctuations of the various densities $p_{l}, p, b$, etc. are calculated by twice differentiating the thermodynamic potential with respect to the appropriate fields. The correlation of the fluctuations of polymer numbers $\delta p_{l} \delta p_{m}=\overline{p_{l} p_{m}}-p_{l} p_{m}$ is

$$
N \overline{\delta p_{l} \delta p_{m}}=-\frac{\delta^{2} f}{\delta \ln \eta_{l} \delta \ln \eta_{m}}=\frac{\delta p_{l}}{\delta \ln \eta_{m}} .
$$

The last derivative on the right-hand side of Eq. (20) is obtained from Eqs. (13) and (16)

$$
N \overline{\delta p_{l} \delta p_{m}}=\delta_{l m} \frac{\eta_{l}}{2 k} x^{l+1}-\frac{\eta_{l} \eta_{m}}{4 k^{2}} x^{l+m+2}(l+1)(m+1)\left(1+\sum_{i=1}^{\infty} \frac{\eta_{i}}{2 k} i(i+1) x^{i+1}\right)^{-1},
$$

where $\delta$ is the Krönecker delta. The fluctuations of the polymers' length $b$ and number $p$ are obtained from Eqs. (11), (14), and (21),

$$
\begin{aligned}
& N \overline{\delta b^{2}}=\sum_{i=1}^{\infty} \frac{\eta_{i}}{2 k} i^{2} x^{i+1}-\left[\sum_{i=1}^{\infty} \frac{\eta_{i}}{2 k} i(i+1) x^{i+1}\right]^{2}\left[1+\sum_{i=1}^{\infty} \frac{\eta_{i}}{2 k} i(i+1) x^{i+1}\right]^{-1}, \\
& N \overline{\delta p^{2}}=\sum_{i=1}^{\infty} \frac{\eta_{i}}{2 k} x^{i+1}-\left[\sum_{i=1}^{\infty} \frac{\eta_{i}}{2 k}(i+1) x^{i+1}\right]^{2}\left[1+\sum_{i=1}^{\infty} \frac{\eta_{i}}{2 k} i(i+1) x^{i+1}\right]^{-1}, \\
& N \overline{\delta p \delta b}=\sum_{i=1}^{\infty} \frac{\eta_{i}}{2 k} i x^{i+1}-\sum_{i=1}^{\infty} \frac{\eta_{i}}{2 k}(i+1) x^{i+1} \sum_{i=1}^{\infty} \frac{\eta_{i}}{2 k} i(i+1) x^{i+1}\left[1+\sum_{i=1}^{\infty} \frac{\eta_{i}}{2 k} i(i+1) x^{i+1}\right]^{-1} .
\end{aligned}
$$




\section{EQUAL REACTIVITY POLYMERIZATION}

In this section we study a special case of the equilibrium polymerization on the equivalent-neighbor lattice: The energy associated with the attachment of one polymer to another does not depend on the sizes of the two polymers. In Flory's terminology ${ }^{11}$ this is equal reactivity. The fugacities $\eta_{l}$ are then all equal to $\eta$. In Sec. III A we determine the dependence of the various thermodynamic quantities on the fugacities $k$ and $\eta$ by specializing the formula derived in Sec. II to the equal reactivity case. In Sec. III B the discrete version of the $n \rightarrow 0$ vector, which was shown by Hillhorst ${ }^{4}$ to possess the same high-temperature expansion as the polymer problem Eq. (1), is solved on the equivalent-neighbor lattice. We verify explicitly the equivalence of the two models in the whole parameter space. It is particularly important to verify this equivalence in exactly soluble models in view of its suggested ${ }^{6}$ breakdown in the low-temperature regime.

\section{A. Thermodynamic functions}

The equation of state Eq. (16) becomes after setting $\eta_{l}=\eta$ for all $l>0$ :

$$
k=x+\eta \frac{x^{2}(2-x)}{2(1-x)^{2}},
$$

where $x=k p_{0}=k \Phi_{0} \leq 1$. For given values of the fields $k$ and $\eta, x$ is determined from the equation of state Eq. (24), and the volume fraction of free monomers (not belonging to polymers) is then obtained, $\Phi_{0}=x / k$. The volume fraction occupied by polymers of $l$ bonds each is, according to Eq. (17),

$$
\Phi_{l}=\frac{\eta}{2 k}(l+1) x^{l+1} \text { for } l>0 .
$$

The total number of polymers per $N$ and the total number of bonds per $N$ are obtained from Eqs. (14) and (15)

$$
\begin{aligned}
& p=\frac{\eta}{2 k} \frac{x^{2}}{1-x}, \\
& b=\frac{\eta}{2 k} \frac{x^{2}}{(1-x)^{2}} .
\end{aligned}
$$

The fluctuations of $p$ and $b$ are calculated from Eqs. (22) and (23)

$$
\begin{aligned}
N \overline{\delta b^{2}}= & \frac{\eta}{2 k} \frac{x^{2}(1+x)}{(1-x)^{3}}\left[1-\frac{\eta}{2 k} \frac{2 x^{2}}{(1-x)^{2}(1+x)}\right) \\
& \times\left(1+\frac{\eta}{2 k} \frac{2 x^{2}}{(1-x)^{3}}\right]^{-1}, \\
N \overline{\delta p^{2}}= & \frac{\eta}{2 k} \frac{x^{2}}{1-x}\left(1-\frac{\eta}{2 k} \frac{x^{2}\left(x^{2}-4 x+2\right)}{(1-x)^{3}}\right) \\
& \times\left(1+\frac{\eta}{2 k} \frac{2 x^{2}}{(1-x)^{3}}\right]^{-1},
\end{aligned}
$$

$$
\begin{aligned}
N \overline{\delta b \delta p}= & \frac{\eta}{2 k} \frac{x^{2}}{(1-x)^{2}}\left[1-\frac{\eta}{2 k} \frac{2 x^{2}}{(1-x)^{2}}\right] \\
& \times\left[1+\frac{\eta}{2 k} \frac{2 x^{2}}{(1-x)^{3}}\right]^{-1} .
\end{aligned}
$$

The equation of state, the thermodynamic quantities, and their fluctuations (measured in scattering experiments) are manifestly singular at $x=1$. From the equation of state Eq. (24), $x=1$ corresponds to $\eta=0$ and $k \geq 1$ which is the phase transitions line in the fields space, and the point $\eta=0, k=1$ is the critical point.

On the $\eta=0$ line the fraction of free monomers $\Phi_{0}$ equals unity as long as $k \leq 1$. For $k>1, \Phi_{0}=1 / k<1$, i.e., some of the monomers are now aggregated into polymers. The total numbers of bonds per $N, b$ is zero if $k \leq 1$ and becomes finite for $k>1, b=(k-1) / k$. The number of polymers per $N$ stays zero for all $k$. Thus the polymerized phase consists of a few macroscopically large polymers. Along the phase transition line $\eta=0$, on both sides of the critical point $k=1$, the following formulas give the thermodynamic quantities as a function of $k$ :

$$
\begin{aligned}
& \Phi_{0}=1 \text { if } k<1 \text { and } \Phi_{0}=1 / k \text { if } k \geq 1, \\
& b=0 \text { if } k<1 \text { and } b=(k-1) / k \text { if } k \geq 1, \\
& p=0 \text { for all } k, \\
& N \overline{\delta b^{2}}=0 \text { if } k<1 \text { and } N \overline{\delta b^{2}}=1 / k \text { if } k \geq 1, \\
& N \overline{\delta p^{2}}=N \overline{\delta b \delta p}=0 \text { for all } k, \\
& f=0 \text { if } k<1 \text { and } f=-\ln k+(k-1) / k \text { if } k \geq 1 .
\end{aligned}
$$

The singular behavior of the thermodynamic quantities along the critical "isotherm" $k=1$ close to the polymerization critical point, $\eta \rightarrow 0$, follows

$$
\begin{aligned}
& \Phi_{0} \approx 1-\left(\frac{1}{2} \eta\right)^{1 / 3}, \quad b \approx\left(\frac{1}{2} \eta\right)^{1 / 3}, \quad p \approx\left(\frac{1}{2} \eta\right)^{2 / 3}, \\
& N \overline{\delta b^{2}} \approx \frac{2}{3}\left[1-\frac{11}{6}\left(\frac{1}{2} \eta\right)^{1 / 3}\right], \quad N \overline{\delta p^{2}} \approx \frac{2}{3}\left(\frac{1}{2} \eta\right)^{2 / 3}, \\
& N \overline{\delta b \delta p} \approx \frac{1}{3}\left(\frac{1}{2} \eta\right)^{1 / 3}, \quad f \approx-\frac{3}{2}\left(\frac{1}{2} \eta\right)^{2 / 3} .
\end{aligned}
$$

\section{B. Polymerization in the magnetic language}

The $O(n)$ vector model in the limit $n \rightarrow 0$ has the same high-temperature series as the polymerization problem, ${ }^{1,2,7,19}$ Eq. (1) with all $\eta_{l}$ equal. Hillhorst ${ }^{4}$ has shown a discrete version of the $O(n)$ model is also equivalent to the equilibrium polymerization. In this subsection we verify the equivalence of Hillhorst's model to the polymerization model on the equivalent-neighbor lattice in the entire parameter space.

Hillhorst's model can be expressed as an Ising-Potts model. At each site $i$ of the lattice there are two variables: $S_{i}= \pm 1$ and $\sigma_{i}=1,2, \ldots, q$. The Hamiltonian is

$-\frac{\mathscr{H}}{k_{B} T}=\frac{J}{2 N} \sum_{i, j} \delta\left(\sigma_{i}, \sigma_{j}\right) S_{i} S_{j}+H \sum_{i} \delta\left(\sigma_{i}, 1\right) S_{i}$

where $\delta$ is the Krönecker delta.

The partition function of this model $Z^{\prime}$ for small $q$, the 
analog of $n \rightarrow 0$, is related to the partition function of the polymerization problem $Z$

$$
Z^{\prime}=\left[2 q\left(1+\frac{1}{2} H^{2}\right)\right]^{N} Z \text {. }
$$

The polymer problem fugacities are related to the magnetic problem fields

$$
k=\frac{J}{1+\frac{1}{2} H^{2}} \text { and } \eta=\frac{H^{2}}{1+\frac{1}{2} H^{2}}
$$

or by inverting Eqs. (35)

$$
J=\frac{k}{1-\frac{1}{2} \eta} \text { and } H^{2}=\frac{\eta}{1-\frac{1}{2} \eta} .
$$

The mapping given in Eqs. (34) and (35), first derived by Gujrati, ${ }^{19}$ differs at high magnetic fields from the mapping used by other authors. ${ }^{1,2}$ The source of this difference is the assignment by the latter of a weight $H^{2} / 2$ to a free monomer. This difference is insignificant close to the polymerized phase, as $H \rightarrow 0$, but nonnegligible at large values of the magnetic field.

Since $k \geq 0$ and $\eta \geq 0$, Eq. (36) implies $J \geq 0$ and $H$ real if $\eta \leq 2$, and $J \leq 0$ and $H$ imaginary if $\eta \geq 2$. Hence if $\eta \leq 2$, the polymerization problem is equivalent to the ferromagnetic $O(n), n \rightarrow 0$, model in a real magnetic field. For $\eta \geq 2$ the polymerization problem is equivalent to the antiferromagnetic $O(n), n \rightarrow 0$, model in an imaginary magnetic field. These statements hold regardless of the underlying lattice.

We now solve the magnetic model defined in Eq. (33). We consider first $J \geq 0$ and $H$ real. By using the Gaussian integral

$\int_{-\infty}^{\infty} \exp \left(-\alpha y^{2}+\beta y\right) d y=\left(\frac{\pi}{\alpha}\right)^{1 / 2} \exp \left(\beta^{2} / 4 \alpha\right), \quad \alpha>0$,

the partition function $Z^{\prime}$ can be written ${ }^{8}$ as

$$
\begin{aligned}
Z^{\prime} & =\sum_{\{\sigma, S\}} \exp \left(-\mathscr{H} / k_{B} T\right) \\
& =\left(\frac{N J}{2 \pi}\right]^{q / 2} \int_{-\infty}^{\infty} \cdots \int_{-\infty}^{\infty} d y_{1}, \ldots, d y_{q} \exp \left(-\frac{1}{2} N J \sum_{m=1}^{q} y_{m}^{2}-N f_{0}\right),
\end{aligned}
$$

where

$$
f_{0}=-\ln \left[2 \cosh \left(\sqrt{q} H+\sqrt{q} J y_{1}\right)+2 \cosh \left(\sqrt{q} J y_{2}\right)+\cdots+2 \cosh \left(\sqrt{q} J y_{q}\right)\right] .
$$

In the thermodynamic limit $N \rightarrow \infty$ the partition function is given by the largest integrand on the right-hand side of Eq. (37), and the free energy $f^{\prime}=-\ln Z^{\prime} / N$ becomes

$$
f^{\prime}=\min _{\left\{y_{1}, \ldots, y_{q}\right\}}\left(\frac{1}{2} J \sum_{m=1}^{q} y_{m}^{2}+f_{0}\right) \text {. }
$$

The optimization equations are

$$
\begin{aligned}
& y_{1}=\frac{\sqrt{q} \sinh \left(\sqrt{q} H+\sqrt{q} J y_{1}\right)}{\cosh \left(\sqrt{q} H+\sqrt{q} J y_{1}\right)+\cosh \left(\sqrt{q} J y_{2}\right)+\cdots+\cosh \left(\sqrt{q} J y_{q}\right)}, \\
& y_{m}=\frac{\sqrt{q} \sinh \left(\sqrt{q} J y_{m}\right)}{\cosh \left(\sqrt{q} H+\sqrt{q} J y_{1}\right)+\cosh \left(\sqrt{q} J y_{2}\right)+\cdots+\cosh \left(\sqrt{q} J y_{q}\right)}, \quad m>1,
\end{aligned}
$$

In the limit $q \rightarrow 0$ for finite $H$, Eqs. (39) and (40) reduce to

$$
f^{\prime}+\ln \left[2 q\left(1+\frac{1}{2} H^{2}\right)\right] \equiv f=\frac{1}{2} J y_{1}^{2}-\ln \left[1+\frac{\left(J y_{1}\right)^{2}}{2\left(1+\frac{1}{2} H^{2}\right)}+\frac{H J y_{1}}{1+\frac{1}{2} H^{2}}\right)
$$

and

$$
y_{1}=\frac{J y_{1}+H}{1+\frac{1}{2}\left(J y_{1}+H\right)^{2}} .
$$

The first part of Eq. (41) is the consequence of Eq. (34). Equation (42) is the mean-field equation of state for the $n \rightarrow 0$ vector model as well as for the Hillhorst model. ${ }^{2,9}$ Our free energy formula Eq. (41) differs from the $f$ of Ref. 2 by an additive factor of $-\ln \left(1+H^{2} / 2\right)$, due to the different weighting of the free monomers. There are more important differences in the large- $\eta$ regime to be discussed next.

If $\eta \geq 2$, Eq. (36) and the positivity of $k$ imply $J \leq 0$ and $H$ imaginary, i.e., $H=i H_{I}$, where $i=\sqrt{-1}, H_{I}$ is real, and $\left|H_{I}\right| \geq \sqrt{2}$. By using the Gaussian integral

$\int_{-\infty}^{\infty} \exp \left(-\alpha y^{2}+i \beta y\right) d y=\left(\frac{\pi}{\alpha}\right)^{1 / 2} \exp \left(-\beta^{2} / 4 \alpha\right), \quad \alpha>0$ and following the same steps as in Eqs. (37)-(42) we find,

$$
f^{\prime}+\ln \left[2 q\left(1-\frac{1}{2} H_{I}^{2}\right)\right] \equiv f,
$$




$$
f=\frac{1}{2}|J| y_{1}^{2}-\ln \left[1+\frac{\left(J y_{1}\right)^{2}}{2\left(\frac{1}{2} H_{I}^{2}-1\right)}+\frac{H_{I}|J| y_{1}}{\frac{1}{2} H_{I}^{2}-1}\right),
$$

where $y_{1}$ is obtained from the equation of state

$$
y_{1}=\frac{|J| y_{1}+H_{I}}{-1+\frac{1}{2}\left(|J| y_{1}+H_{I}\right)^{2}} .
$$

We verify the equivalence of the magnetic language results to the polymerization problem results of Sec. III A for $J \geq 0$ and real $H$ or $\eta \leq 2$. The same procedure can be used for negative $J$ and imaginary $H$ or $\eta \geq 2$. Applying the mapping given in Eq. (35), the number of bonds per $N$ is

$$
b=-k\left(\frac{\delta f}{\delta k}\right)_{\eta}=-J\left(\frac{\delta f}{\delta J}\right)_{H}=\frac{1}{2} J y_{1}^{2},
$$

where the last part of Eq. (45) was obtained by differentiating the right-hand side of Eq. (41) and by using Eq. (42). By comparing the above formula to Eq. (27), derived in Sec. III A for $b$, we find

$$
\Phi_{0}=\frac{y_{1}\left(1+\frac{1}{2} H^{2}\right)}{H+J y_{1}}
$$

where $\Phi_{0}$ is the fraction of free monomers. It follows from the equation of state (42) and Eq. (46) that

$$
1+\frac{\left(J y_{1}\right)^{2}}{2\left(1+\frac{1}{2} H^{2}\right)}+\frac{H J y_{1}}{1+\frac{1}{2} H^{2}}=\Phi_{0}^{-1} .
$$

Equations (45) and (47) allow us to rewrite the free energy from Eq. (41) as $f=\ln \Phi_{0}+b$, the general expression for the thermodynamic potential of the polymerization problem, Eq. (19). The equation of state (24) is identical to the equation of state in the magnetic language Eq. (42), reached by using the mapping given in Eqs. (35) and (46). This completes the verification of the equivalence of the $q \rightarrow 0$ Ising-Potts model, or the Hillhorst model, to equilibrium polymerization for the entire parameter space.

\section{THE SCHULZ DISTRIBUTION}

Flory ${ }^{11}$ argued that the exponential decay of the number of polymers of a given size as a function of the polymer size, the so-called most probable distribution, is implied by the equal reactivity principle. Accordingly, the opportunity for reaction between two aggregates is always the same, regardless of the aggregates' sizes. In our notation this is equivalent to $\eta_{l}=\eta$ for all $l$. However, certain polymerization processes exhibit a dependence of the number of polymers of given size on the polymer size better fitted by a gamma distribution, exponential times power, the so-called Schulz distribution. ${ }^{12}$ Within our model of equilibrium polymerization on the equivalentneighbor lattice, Sec. III, the Schulz distribution can be achieved by relaxing Flory's equal reactivity principle, assuming a power dependence on the polymer size for the fugacities $\eta_{l}=\eta(l+1)^{r-1}$, where $r$ is a real number we call the Schulz index. For $r=1$ we recover the equal reactivity polymerization.
The thermodynamic quantities are calculated by specializing the general formula of Sec. II to the power dependence of the fugacities. The number of polymers of $l$ bonds each per $N, p_{l}$, and the volume fraction occupied by these polymers are obtained from Eqs. (13) and (17),

$$
\begin{aligned}
& p_{l}=\frac{\eta}{2 k} x^{l+1}(l+1)^{r-1}, \quad l=1,2,3, \ldots, \\
& \Phi_{l}=\frac{\eta}{2 k} x^{l+1}(l+1)^{r}, \quad l=1,2,3, \ldots,
\end{aligned}
$$

and

$$
p_{0}=\Phi_{0}=x / k \text {. }
$$

The total number of polymers and the total number of chemical bonds per $N$ are obtained from Eqs. (14) and (15),

$$
\begin{aligned}
& p=\frac{\eta}{2 k}\left[E_{r-1}(x)-x\right], \\
& b=\frac{\eta}{2 k}\left[E_{r}(x)-E_{r-1}(x)\right],
\end{aligned}
$$

where $E_{r}(x)$ stands for

$$
E_{r}(x)=\sum_{n=1}^{\infty} n^{r} x^{n}
$$

For given values of the fields $k$ and $\eta$, the parameter $x$ is determined by the equation of state $\Phi_{0}+b+p=1$, which in view of Eqs. (50) - (52) is,

$$
k=x+\frac{1}{2} \eta\left[E_{r}(x)-x\right] \text {. }
$$

The various thermodynamic quantities are then calculated from Eqs. (48) $-(52)$. The thermodynamic potential is determined by Eq. (19), $f=\ln \Phi_{0}+b$.

The function $E_{r}(x)$ defined in Eq. (53) appears in the theory of the ideal Bose-Einstein gas, whose analytical properties are well researched. ${ }^{20}$ For $x<1, E_{r}(x)$ is finite, while for $x>1$ the series on the right-hand side of Eq. (53) diverges. For $x=1, \quad E_{r}(1)=Z(-r)$, the Riemann $\zeta$ function, which is finite if $r<-1$ and infinite if $r \geq-1$. For any value of the index $r, x=1$ is a singular point. For small $1-x$ the leading singular contribution to $E_{r}$ is $\Gamma(r+1)(1-x)^{-(r+1)}$, where $\Gamma$ is the gamma function. For $r=-1,-2, \ldots$ the leading singularity is modified by a logarithm $(1-x)^{-(r+1)} \ln (1-x)$.

The locus of points in the fields space $k, \eta$ where the thermodynamic quantities exhibit nonanalytical behavior is determined by setting $x=1$ in the equation of state (54),

$$
\begin{aligned}
& \eta=0, \quad k_{c}=1 \text { if } r \geq-1, \\
& k_{c}=1+\frac{1}{2} \eta[Z(-r)-1] \text { if } r<-1 .
\end{aligned}
$$

If the fugacity $k$ is larger than the critical value $k_{c}$, infinitely large polymers are formed and thus $k_{c}(\eta)$ represents the critical polymerization locus.

The nonanalytic behavior of thermodynamic quantities close to the critical polymerization locus is determined next. We start with $r \geq-1$. Along the $\eta=0$ line we find 


$$
b=0 \text { if } k \leq 1 \text { and } b=(k-1) / k \text { if } k \geq 1
$$

and

$$
p=0 \text { for any } k \text {. }
$$

Thus for $\eta=0$ and $k>k_{c}=1$, a small number $(p=0)$ of infinitely large polymers $(b=$ finite) is formed. The thermodynamic potential $f$, for $\eta=0$, is

$$
f=0 \text { if } k<1
$$

and

$$
f=-\ln k+(k-1) / k \text { if } k \geq 1 .
$$

For $k=1$, the "critical isotherm," as $\eta$ approaches zero, we find

$$
\begin{aligned}
f \approx & -\frac{r+2}{2 r}\left[\frac{1}{2} \Gamma(r+1) \eta\right]^{2 /(r+2)}+O(\eta) \text { if } r>0, \\
f \approx & \frac{1}{4} \eta \ln \eta+O(\eta) \text { if } r=0, \\
f \approx & -\frac{1}{2}[Z(-r+1)-1] \eta \\
& \quad+\frac{r+2}{2 r}\left[\frac{1}{2} \Gamma(r+1) \eta\right]^{2 /(r+2)}+O\left(\eta^{2}\right) \text { if }-1<r<0, \\
f \approx & -\frac{1}{2}[Z(2)-1] \eta+\frac{1}{2}\left(\frac{1}{2} \eta\right)^{2}\left[\ln \left(\frac{1}{2} \eta\right)\right]^{2} \text { if } r=-1,
\end{aligned}
$$

where $Z$ stands for the Riemann $\zeta$ function and $\Gamma$ stands for the gamma function. Equation (59) with $r=1$ reduces to Eq. (32). The behavior of $f$ along the $\eta=0$ and $k=1$ paths is consistent with the following scaling representation of the singular part of the thermodynamic potential $f_{s}$,

$$
f_{s}=|k-1|^{2-\alpha} A_{ \pm}\left(\eta /|k-1|^{\Delta}\right),
$$

where $\alpha=0$ and $\Delta=r+2$ for $r \geq-1$. Thus the universality class of equilibrium polymerization changes continuously with the Schulz index $r$. Note the logarithmic modifications ${ }^{21}$ of the power law when the Schulz index $r$ equals -1 and 0 .

Now consider $r<-1$. The values of the thermodynamic quantities on the critical polymerization line $k_{c}(\eta)$ are obtained by setting $x=1$ in the appropriate equations,

$$
\begin{aligned}
& k_{c}=1+\frac{1}{2} \eta[Z(-r)-1], \\
& b_{c}=\frac{\eta}{2 k_{c}}[Z(-r)-Z(-r+1)], \\
& p_{c}=\frac{\eta}{2 k_{c}}[Z(-r+1)-1], \\
& \Phi_{0 c}=1 / k_{c}, \\
& N \overline{\delta b^{2}}=1-b_{c}, \\
& N \overline{\delta p^{2}}=-N \overline{\delta b \delta p}=p_{c} .
\end{aligned}
$$

When approaching the critical polymerization from the high-temperature side, i.e., $k<k_{c}$, the thermodynamic quantities exhibit singular behavior determined by the nonanalyticity of the function $E_{r}(x)$, defined in Eq. (53), at $x=1$. For $-2<r<-1$ the leading nonanalytic con- tributions to $b$ and $p$ are proportional to $\left(k_{c}-k\right)^{1 /(-r-1)}$. When the Schulz index is even smaller $r<-2$, the leading nonanalytic contributions to $b$ and $p$ are proportional to $\left(k_{c}-k\right)^{-r-1}$. If the Schulz index $r=-2,-3,-4, \ldots$, the power-law singularity is modified by logarithms. ${ }^{21}$ For example, if $r=-2$ the leading singularity of $b$ or $p$ is proportional to $\left(k_{c}-k\right) / \ln \left(k_{c}-k\right)$.

What happens for $r<-1$ in the low-temperature regime $\left(k \geq k_{c}\right)$ ? Since $E_{r}(x)-x$ is a monotonically increasing function of $x$, the equation of state Eq. (54) does not have a solution for

$$
k \geq k_{c}=1+\frac{1}{2} \eta[Z(-r)-1] .
$$

Though a careful analysis of the thermodynamic limit is yet to be performed, we expect in this regime a BoseEinstein condensationlike phenomenon. The volume fractions occupied by finite polymers stay at their largest possible values, which in view of Eqs. (49) and (50) are achieved for $x=1$,

$$
\begin{aligned}
& \Phi_{0}=1 / k, \\
& \Phi_{l}=\frac{\eta}{2 k}(l+1)^{r} .
\end{aligned}
$$

A finite-volume fraction is now occupied by infinitely large polymers $\Phi_{\infty}$. The equation of state Eq. (54) must then be replaced by

$$
\sum_{l=0}^{\infty} \Phi_{l}+\Phi_{\infty}=1
$$

which after using Eqs. (61) and (62) becomes

$$
\Phi_{\infty}=\left(k-k_{c}\right) / k \text {. }
$$

The number of polymers per $N$ and the number of bonds per $N$ are

$$
\begin{aligned}
p & =\sum_{l=1}^{\infty} \Phi_{l} /(l+1)=\frac{\eta}{2 k}[Z(-r+1)-1], \\
b & =\sum_{l=1}^{\infty} l \Phi_{l} /(l+1)+\Phi_{\infty} \\
& =1-\frac{1}{k}-\frac{\eta}{2 k}[Z(-r+1)-1] .
\end{aligned}
$$

The fluctuations of $b$ and $p$ are obtained by differentiating $b$ and $p$ with respect to $\ln k$ and $\ln \eta$,

$$
N \overline{\delta b^{2}}=1-b, \quad N \overline{\delta p^{2}}=-N \overline{\delta b \delta p}=p .
$$

The thermodynamic potential $f$ is obtained by substituting on the right-hand side of Eq. (19) the expressions for $\Phi_{0}$ and $b$ from Eqs. (62) and (64),

$$
f=1-\frac{1}{k}-\frac{\eta}{2 k}[Z(-r+1)-1]-\ln k
$$

An important difference between the polymerized phases for $r \geq-1$ and $r<-1$, respectively, is that in the former there are a few macroscopically large polymers, $p=0, b=$ finite, while in the latter the polymerized material consists of a large number ( $p_{l}$ is finite) of finitely 
large and a few $\left(p_{\infty}=0\right)$ infinitely large polymers. The latter is reminiscent of the two-fluid picture of superfluidity. ${ }^{13}$ The mathematical analogy between polymers of Schulz index $r$ and the Bose-Einstein ideal gas in $d$ dimensions is borne by the function $E_{r}$, with $r=-d / 2$. The critical values of the Schulz index $r=-1$ and -2 correspond to the lower and upper critical dimensions for Bose condensation $d=2$ and 4 , respectively.

\section{SUMMARY}

We have presented the exact solution of the linear polymerization process on the equivalent-neighbor lattice [ $N$ vertices and $\frac{1}{2} N(N-1)$ edges connecting all pairs of vertices]. Other exact solutions for the polymerization process in the literature concern the one-dimensional ${ }^{7}$ and the Bethe lattice (interior of Cayley tree) ${ }^{22}$ problems. Exact solutions are desirable because a large proportion of the studies on the polymerization problem have used its high-temperature mapping into the $n \rightarrow 0$ vector model, and the validity of the mapping at low temperatures was questioned by Gujrati. ${ }^{5,6}$ In this connection Wheeler et $a .^{3}$ have discussed the influence of the order of limits $N \rightarrow \infty, n \rightarrow 0$, and $H \rightarrow 0$ on the existence of the polymerized phase. In Sec. III we have verified that if the order of the limits is as above (from left to right) the discrete version of the vector model provides the correct solution of the polymerization process at all temperatures. Since this verification was performed for the equivalent-neighbor lattice, a model suitable for high dimensions, we cannot rule out peculiarities of the type discussed by Gujrati ${ }^{5,6}$ in three-dimensional systems.

The polymerization process analyzed in Sec. II is more general than the one discussed by other authors ${ }^{1-7,9-11,19,22}$ in the sense that the fugacity controlling the number of polymers is allowed to depend on polymers sizes. This generalization enables rich critical po- lymerization behavior as explicitly demonstrated in Sec. IV, for the special case of a power dependence of the fugacity $\eta_{l}$ on the polymer size $\eta_{l}=\eta(l+1)^{r-1}$. Polymers sizes have a gamma distribution in this case, also known in the polymer literature as the Schulz distribution. The critical exponents vary continuously ${ }^{23}$ with the Schulz index $r$. For $r \geq-1$ the phase diagram is identical to the one obtained for $r=1$, which corresponds to the equal reactivity case of $\eta_{l}=\eta$ for all $l$. The polymerized phase appears at low enough temperatures $k>1$, in the limit $\eta \rightarrow 0$. If $r<-1$, on the other hand, a critical line $k=k_{c}(\eta)$ separates the polymerized and the unpolymerized phases. There is a close mathematical analogy between the polymerization process of index $r$ and the Bose-Einstein ideal gas in $d=-2 r$ dimensions. For instance, the critical values $r=-1$ and -2 correspond to the lower and upper critical dimensions for Bose-Einstein condensation $d=2$ and 4 , respectively. The equalreactivity polymerization $r=1$ corresponds to the BoseEinstein gas in $d=-2$ dimensions.

The entropy associated with polymers located on the equivalent-neighbor lattice is equal ${ }^{24}$ to the FloryHuggins entropy of mixing ${ }^{15}$ plus the disorientation entropy. ${ }^{16}$ This observation offers the opportunity to render rigorous the large number of thermodynamic studies $^{25}$ (micellar mixtures, polymers, etc.) where the FloryHuggins theory is applied.

\section{ACKNOWLEDGMENTS}

This work was supported by NASA Grant No. 2140460359 and by the Ohio Board of Regents. Some of the work was performed during a visit with Mehran Kardar at the Massachusetts Institute of Technology (MIT). I am grateful for his hospitality and support through $\mathrm{Na}$ tional Science Foundation (NSF) Grant No. DMR-8620386. The author thanks Puru Gujrati and Mehran Kardar for helpful discussions.
${ }^{1}$ P. G. DeGennes, Phys. Lett. 38A, 339 (1972); J. des Cloiseaux, J. Phys. 36, 281 (1975).

2J. C. Wheeler and P. Pfeuty, Phys. Rev. A 24, 1050 (1981).

${ }^{3}$ J. C. Wheeler, J. F. Stilck, R. G. Petschek, and P. Pfeuty, Phys. Rev. B 35, 284 (1987).

${ }^{4}$ H. J. Hillhorst, Phys. Rev. B 16, 1253 (1977).

${ }^{5}$ P. D. Gujrati, Phys. Rev. B 31, 4375 (1985). See, however, Ref. 3.

${ }^{6}$ P. D. Gujrati, Phys. Rev. B 35, 8486 (1987).

${ }^{7}$ P. Pfeuty and J. C. Wheeler, Phys. Rev. A 27, 2178 (1983).

${ }^{8}$ M. Kardar, Phys. Rev. Lett. 51, 523 (1983); Phys. Rev. B 28, 244 (1983).

${ }^{9}$ P. D. Gujrati, Phys. Rev. B 25, 3381 (1982).

${ }^{10}$ A. V. Tobolsky and A. Eisenberg, J. Am. Chem. Soc. 81, 7810 (1959).

${ }^{11}$ J. Flory, Principles of Polymer Chemistry (Cornell University Press, Ithaca, 1986), p. 319.

${ }^{12}$ G. V. Schulz, Z. Phys. Chem. B 43, 25 (1939); M. Kurata, Thermodynamics of Polymer Solutions (Academic, New York, 1982), Vol. 1. Other distributions, e.g., normal-logarithmic investigated by K. Solk, Macromolecules 8, 819 (1975), could also have experimental relevance.

${ }^{13}$ L. D. Landau, E. M. Lifshitz, and L. P. Pitaevskii, Course of Theoretical Physics: Statistical Physics (Pèrgamon, New York, 1980), Vol. 9, Part 2, pp. 88-93.

${ }^{14}$ H. Jacobson and W. H. Stockmayer, J. Chem. Phys. 18, 1600 (1950); H. Jacobson, C. O. Beckmann, and W. H. Stockmayer, ibid. 18, 1607 (1950); R. G. Petschek, P. Pfeuty, and J. C. Wheeler, Phys. Rev. A 34, 2391 (1986).

${ }^{15}$ P. J. Flory, Ref. 11, pp. 502 and 503.

${ }^{16}$ There are some slight departures (to be discussed elsewhere) from Flory's formula for the disorientation entropy [see Ref. 15, Eq. (9)].

${ }^{17}$ M. Kaufman and R. B. Griffiths, Phys. Rev. B 28, 3864 (1983) give examples which show why it is desirable for an approximate method to be realizable [in the sense of A. N. Berker and S. Ostlund, J. Phys. C 12, 4961 (1979)].

${ }^{18}$ R. J. Baxter, Exactly Solved Models in Statistical Mechanics (Academic, New York, 1982), p. 40.

${ }^{19}$ P. D. Gujrati, Phys. Rev. A 24, 2096 (1981).

${ }^{20}$ R. K. Pathria, Statistical Mechanics (Pergamon, New York, 1972), pp. 503 and 504. 
${ }^{21}$ M. Kaufman and D. Andelman, Phys. Rev. B 29, 4010 (1984); S. I. Chase and M. Kaufman, ibid. 33, 239 (1986); M. Kaufman, ibid. 36, 3697 (1987).

22J. F. Stilck and J. C. Wheeler, J. Stat. Phys. 46, 1 (1987); P. D. Gujrati, Phys. Rev. Lett. 53, 2453 (1984).

${ }^{23}$ There are other equivalent-neighbor interaction models exhibiting continuously varying exponents: $\mathbf{M}$. Kardar and $\mathbf{M}$. Kaufman, Phys. Rev. Lett. 51, 1210 (1983); M. Kaufman and
M. Kardar, Phys. Rev. B 30, 1609 (1984); M. Kaufman and M. Kahana, ibid. 37, 7638 (1988).

${ }^{24}$ See also R. Patil, thesis, University of Akron, OH, 1988.

${ }^{25}$ See, for example, D. Blankschtein, G. M. Thurston, and G. B. Benedek, J. Chem. Phys. 85, 7268 (1986); R. Goldstein, ibid. 84, 3367 (1986); K. Solk, L. A. Kleintjens, and R. Königsveld, Macromolecules 17, 573 (1984). 